\title{
Comparison of the antimicrobial and hemolytic
} activities of various forms of silver (ions, nanoparticles, bioconjugates) stabilized in a zeolite

\section{matrix}

Olga Yu. Golubeva $a^{* a}$, Natalia Yu. Ulyanova $a^{a}$ Elizaveta V. Vladimirova ${ }^{b}$, Olga V. Shamova ${ }^{b}$

${ }^{a}$ Institute of Silicate Chemistry, Russian Academy of Sciences, Adm. Makarova Emb., 2,St. Petersburg, 199034, Russia

b Institute of Experimental Medicine, Academic Pavlov Str. 12, St.Petersburg, 197376, Russia

\section{Table of Contents}

1. Physical and chemical research methods detailed description S2

2. Antimicrobial activity measurements description $\mathbf{S 3}$

3. Hemolytic activity tests description $\quad$ S4

4 FTIR results $\quad$ S5

Fig. S1. FTIR spectra of lysozyme, $\mathrm{Ag} / \mathrm{Lyz}$ nanoparticles and Beta/Ag ${ }^{\circ}-\quad$ S5 Lyz- $0.8 \%$.

5. $\mathrm{N}_{2}$ adsorption-desorption isotherms of the samples

Fig. S2. $\mathrm{N}_{2}$ adsorption-desorption isotherms of the samples: $a$-zeolite Beta; $b$ - Beta $/ \mathrm{Ag}^{\circ}$-Lyz- $0.8 \%, c$ - Beta/ $/ \mathrm{Ag}^{+}-5 \%, d$ - Beta $/ \mathrm{Ag}^{\circ}-5 \%$. 


\section{Physical and chemical research methods detained description}

The X-ray diffraction patterns of the samples were determined using a Bruker D8Advantage powder diffractometer (BRUKER AXS GmbH, Karlsruhe, Germany, CuKa-radiation range; tube operation mode $40 \mathrm{~kW} / 40 \mathrm{~mA}$; Vantec-1 position-sensitive detector; $\theta$ - $\theta$-geometry; range $2 \theta 5-70^{\circ}$, step $2 \theta=0.0224^{\circ}$; intensity of the maximum peak $\sim 283000 \mathrm{imp}$ ).

The UV adsorption spectra were carried out on a UV-vis spectrometer with an integrating sphere (Shimadzu UV-2600, Germany). Silver nanoparticle sols were preliminary diluted 10 times and measured in quartz cuvettes with the optical path length of $5 \mathrm{~mm}$. The absorption spectra of Beta/ $\mathrm{Ag}^{o}$-Lyz powders were out using tableted samples mixed with $\mathrm{BaSO}_{4}$.

Chemical analysis of samples of zeolite matrices for the content of Si and Al was carried out by gravimetric method using quinolate of silicon-molybdenum complex and by complexometric titration. The content of silver and alkali cations was determined by flame photometry on an iCE3000 atomic absorption spectrometer (Thermo scientific iCE 3000, USA). The error in determining the silver content was $1-3 \%$ of the measured value.

The $\zeta$-potential of the samples was determined using the particle size and zeta potential analyser NaniBrook 90 PlusZeta (Brookehaven Instruments Corporation, USA).

The textural parameters of the materials were determined by means of low-temperature adsorption-desorption of nitrogen. The isotherms were collected using a Quantachrome NOVA 1200e instrument (Quantachrome Instruments, Boynton Beach, USA). Degassing was performed at $300{ }^{\circ} \mathrm{C}$ for $12 \mathrm{~h}$. The specific surface area of the sample was calculated by the BET method (Brunauer, S.; Emmett, P. H.; Teller, E. Adsorption of Gases in Multimolecular Layers. J. Am. Chem. Soc. 1938, 60 (2), 309-319. https://doi.org/10.1021/ja01269a023.) Pore size distribution was calculated by the Barrett-Joyner-Halenda method (BJH) (Barrett, E. P.; Joyner, L. G.; Halenda, P. P. The Determination of Pore Volume and Area Distributions in Porous Substances. I. Computations from Nitrogen Isotherms. J. Am. Chem. Soc. 1951, 73 (1), 373-380. https://doi.org/10.1021/ja01145a126.) и Density Function Theory method (DFT). The experimental data obtained (analysis of pore sizes and their size distribution) were processed using the NOVAWin software. The error in determining the specific surface area was $1 \%$. The weighed portions of the test samples in the form of a powder were $0.1 \pm 0.02 \mathrm{~g}$.

The morphology of the samples was studied using scanning electron microscopy (SEM) on an FEI Quanta Inspect (USA) and Tescan MIRA 3 LMU (Czech Republic) instrument using a standard sample preparation method. In order to determine the silver nanoparticles and their distribution over zeolite surface, an R-BSE detector of reflected electrons of the scintillation type was used. Transmission electron microscopy (TEM) studies were carried out using high- 
resolution electron microscope JEM 2100-F (Japan), 200 kV FE. Samples were prepared using a standard carbon replica technique.

\section{Antimicrobial activity measurements description}

Antimicrobial activity of the samples was assessed by broth microdilution assay (Methods in Molecular Biology. Volume 78. Antibacterial Peptide Protocols Edited by William S. Shafer. The Humana Press, Totowa, NJ. 1997. x + 259 Pp. $15.5 \times 23.5$ Cm. ISBN 0-89603-408-9. \$74.50. J. Med. Chem. 1997, 40 (25), 4161. https://doi.org/10.1021/jm970565e.) against a drugresistant clinical isolate of Gram-positive bacteria Staphylococcus aureus 1399/17 (resistant to the following antibiotics: ampicillin, oxacillin, gentamicin, amikacin, ofloxacin), as well as against Gram-negative bacteria Pseudomonas aeruginosa 522/17 MDR (resistant to meropenem, ceftazidime, cefixime, amikacin, gentamycin, netilmicin, ciprofloxacin, colistin), Klebsiella pneumoniae ESBL 344 (extended spectrum beta-lactamases, resistant to ampicillin and other penicillins) (the microorganisms were kindly provided by Dr. A. Afinogenova, Institute of Epidemiology and Microbiology named after L. Pasteur, Russia) and Escherichia coli ML-35p (ampicillin-resistant strain, provided by Professor R. Lehrer, University of California, Los Angeles, USA).

In the experiments, we used cultures of bacteria in the logarithmic growth phase with a concentration of $1 \times 10^{5}$ colony-forming units (CFU) per ml. The bacteria were grown in $2.1 \%$ Muller-Hinton broth M391 (HiMedia, India).

Two-fold serial dilutions of the test samples were carried out in $0.01 \mathrm{M}$ sodium phosphate buffer pH 7.4 containing $0.1 \%$ bovine serum albumin and added in a volume of $5 \mu \mathrm{L}$ to wells of sterile 60-well Terasaki microplates. An equivalent amount of the bacterial suspension was added to the same wells. The control samples were the following: a sample containing only the medium as proof of sterility, and a sample containing bacteria without the tested substances as a control of bacterial viability. The microplates were incubated at $37^{\circ} \mathrm{C}$ for $18-20 \mathrm{~h}$.

The minimum inhibitory concentration (MIC) was taken as the highest concentration of the substance at which the visible growth of microorganisms was not observed in the corresponding wells of the microplate. 


\section{Hemolytic activity tests description}

A hemolytic test was used to determine the ability of the studied samples to damage membranes of eukaryotic cells. Human erythrocytes obtained from peripheral blood of healthy donors by a standard procedure (Methods in Molecular Biology. Volume 78. Antibacterial Peptide Protocols Edited by William S. Shafer. The Humana Press, Totowa, NJ. 1997. x + 259 Pp. $15.5 \times 23.5$ Cm. ISBN 0-89603-408-9. \$74.50. J. Med. Chem. 1997, 40 (25), 4161. https://doi.org/10.1021/jm970565e) were used to determine the hemolytic activity. The serial dilutions of the analyzed samples were prepared in a range of concentrations from $0.16 \mathrm{mg} / \mathrm{ml}$ to $10 \mathrm{mg} / \mathrm{ml}$ in $0.01 \%$ acetic acid. $10 \mu \mathrm{L}$ of each experimental sample was added to $90 \mu \mathrm{L}$ of a $2.8 \%$ suspension of erythrocytes. After adding the samples, $2.5 \%$ concentration of the erythrocyte suspensions was achieved.

To obtain a positive control (100\% erythrocyte lysis), $10 \mu \mathrm{L}$ of Triton X-100 detergent diluted 10 times in an aqueous solution of $0.01 \%$ acetic acid was added to $90 \mu \mathrm{L}$ of the erythrocyte suspension. For a negative control ( $0 \%$ erythrocyte lysis) $10 \mu \mathrm{L}$ of $0.01 \%$ acetic acid was added to $90 \mu \mathrm{L}$ of the erythrocyte suspension. Analyzed solutions ( 3 parallels for each of the studied samples) were incubated at $37{ }^{\circ} \mathrm{C}$ for $30 \mathrm{~min}$. Then the samples were centrifuged at $10,000 \mathrm{~g}$ for $4 \mathrm{~min}$. Supernatant $(200 \mu \mathrm{L})$ of each sample was taken and added to the wells of a 96-well plate (Costar, Corning Inc.). The optical density of the samples was measured using a SpectraMax 250 spectrophotometer (Molecular Devices, Sunnyvale, USA) at a wavelength of $540 \mathrm{~nm}$ to judge the content of hemoglobin released from destroyed erythrocytes.

The percentage of hemolysis was calculated using the formula:

Hemolysis $(\%)=\left(\left(\mathrm{OD}_{540}(\right.\right.$ sample $)-\mathrm{OD}_{540}(0 \%$ lysis $\left.)\right) /\left(\mathrm{OD}_{540}(100 \%\right.$ lysis $)-\mathrm{OD}_{540}(0 \%$ lysis $\left.)\right)$ x $100 \%$

The data are presented as means and standard deviations from the results obtained by three independent experiments, where the experimental and control samples were prepared in triplicates. 


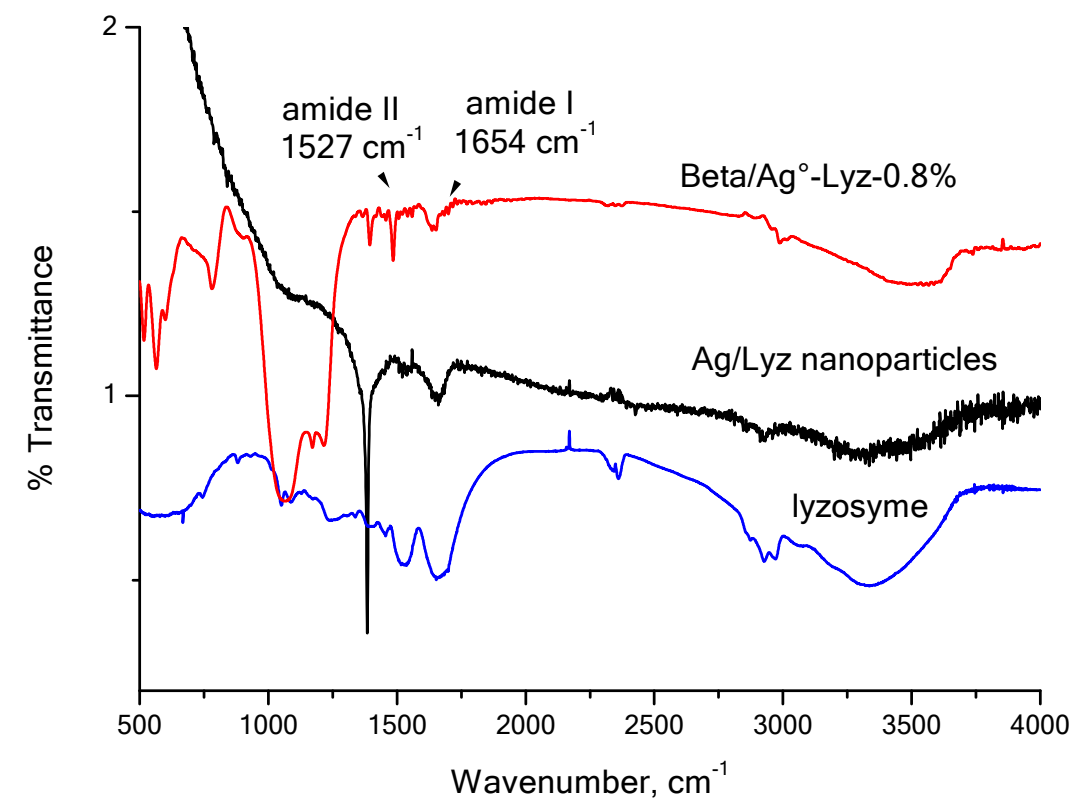

Figure S1. Comparison of FTIR spectra of lysozyme, Ag/Lyz nanoparticles and Beta/ $\mathrm{Ag}^{\circ}-\mathrm{Lyz}-$ $0.8 \%$.

The FTIR spectra of Beta $/ \mathrm{Ag}^{\circ}$-Lyz- $0.8 \%$ contain characteristic bands associated with the stretching of the $\mathrm{C}=\mathrm{O}$ bond (1640-1660 $\mathrm{cm}^{-1}$, amide I band) and deformation of the $\mathrm{N}-\mathrm{H}$ bond $\left(1520-1550 \mathrm{~cm}^{-1}\right.$, amide II band). 
The porous structure was analysed based on the low-temperature nitrogen sorption method (Quantachrome NOVA 1200e, USA; relative pressure range: 0.005-0.999 $\mathrm{P} / \mathrm{P}_{\mathrm{o}}$, pressure resolution $0.0015 \%$ ). Degassing was performed at $300{ }^{\circ} \mathrm{C}$ for $12 \mathrm{~h}$. The specific surface area (SSA) of the sample was calculated by the BET method using NOVAWin (USA) software. The relative error in the specific surface area value was $1 \%$.

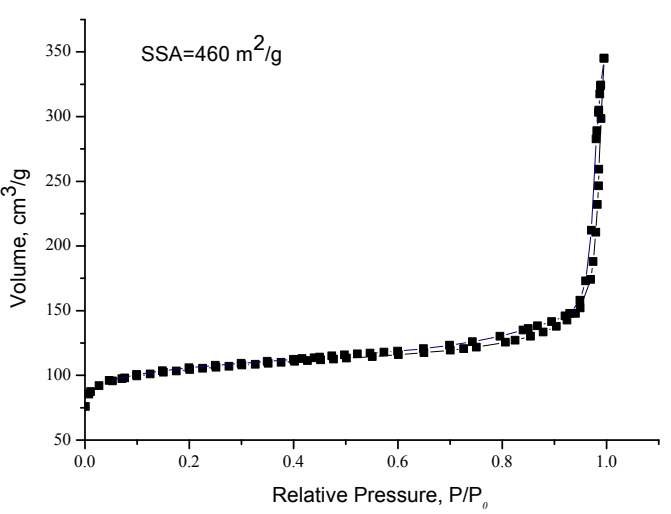

$a$

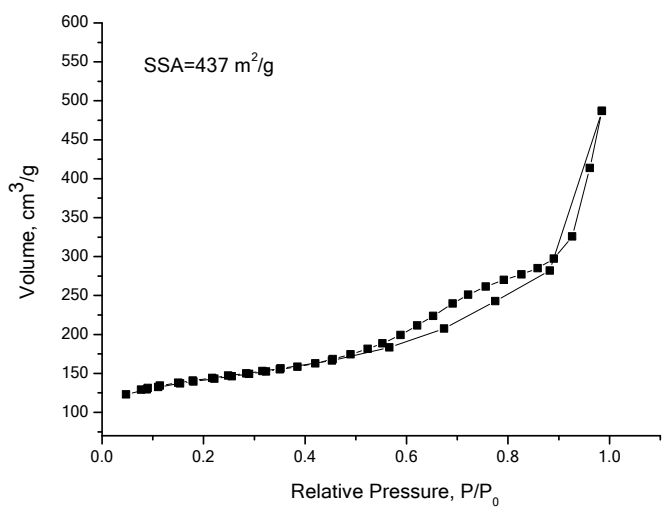

C

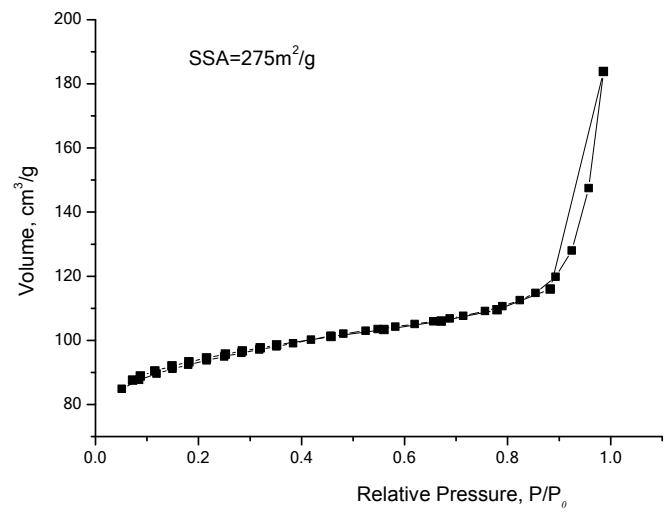

$b$

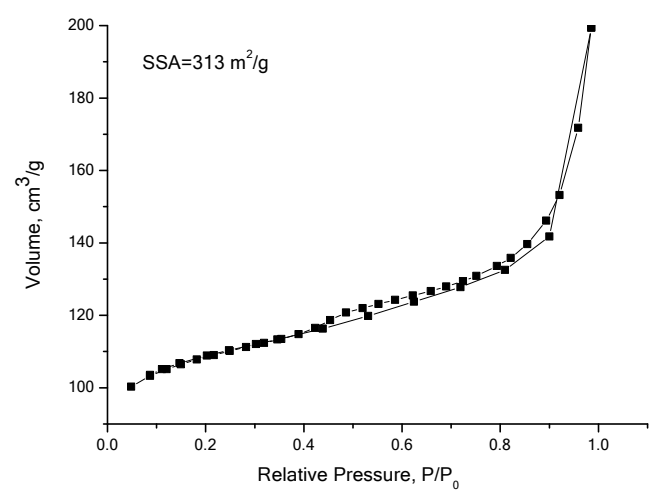

$d$

Figure S2. $\mathrm{N}_{2}$ adsorption-desorption isotherms of the samples: $a$-zeolite Beta; $b-\mathrm{Beta} / \mathrm{Ag}^{\circ}$ -

$$
\text { Lyz- } 0.8 \%, c-\text { Beta } / \mathrm{Ag}^{+}-5 \%, d-\operatorname{Beta} / \mathrm{Ag}^{\circ}-5 \% \text {. }
$$

A decrease in the specific surface area is observed for zeolite beta samples modified with ions, nanoparticles, and bioconjugates, which is apparently associated with the clogging of the cavities of the zeolite as a result of its modification with silver ions and nanoparticles and the impossibility of $\mathrm{N}_{2}$ adsorption during the surface area measurement. 